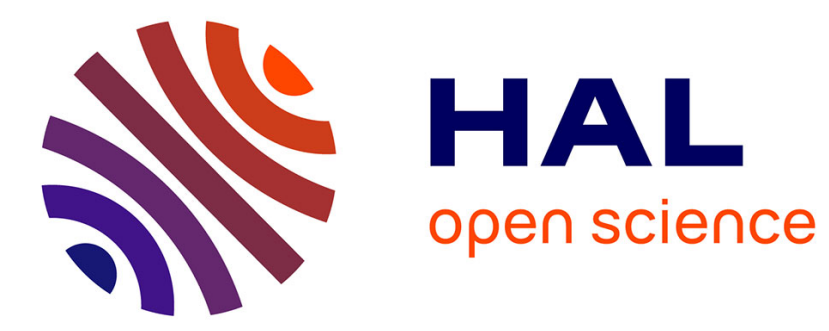

\title{
Referral hiring and labor markets: a computational study
}

Samuel Thiriot, Zach Lewkovicz, Philippe Caillou, Jean-Daniel Kant

\section{To cite this version:}

Samuel Thiriot, Zach Lewkovicz, Philippe Caillou, Jean-Daniel Kant. Referral hiring and labor markets: a computational study. Artificial Economics 2011, Sep 2011, The Hague, Netherlands. pp.15-25, 10.1007/978-3-642-21108-9_2 . inria-00579625

\section{HAL Id: inria-00579625 https://hal.inria.fr/inria-00579625}

Submitted on 24 Nov 2011

HAL is a multi-disciplinary open access archive for the deposit and dissemination of scientific research documents, whether they are published or not. The documents may come from teaching and research institutions in France or abroad, or from public or private research centers.
L'archive ouverte pluridisciplinaire HAL, est destinée au dépôt et à la diffusion de documents scientifiques de niveau recherche, publiés ou non, émanant des établissements d'enseignement et de recherche français ou étrangers, des laboratoires publics ou privés. 


\title{
Referral hiring and labor markets: a computational study
}

\author{
Samuel Thiriot, Zach Lewkovicz, Philippe Caillou and Jean-Daniel Kant
}

\begin{abstract}
Many empirical studies emphasize the role of social networks in job search. The social network implicated in this process is known to be characterized by diversified properties, including communities, homophily or ties having various strength levels. Nevertheless, previous models of the labor markets fail to capture the complexity of social networks, as each specific network requires the development of specific algorithms. In this paper, we rather rely on an independent generic network generator for creating detailed networks describing friendships, colleagues, communities and various degrees of connectivity. We build a simple model of the labor market in which individuals find positions solely through their acquaintances, and update their network when being hired. This original experimental setting facilitates the analysis of various characteristics of networks in the labor market, including various sizes, the number of friendship links or the impact of communities. Experiments confirm the "strength of weak ties" phenomenon. However, the initial characteristics of the network like the existence of communities are shown to be destroyed by the implausible mechanisms integrated into this simplistic model; this suggests that the impact of plausible networks on models' dynamics may only be studied when the mechanisms of this economic model are plausible as well - in other words, "a model is only as descriptive as its most implausible component". Experiments show that, whereas friend are always more useful than colleagues, weak friends are especially useful when the market is stable and with low unemployment and vacancy rate.
\end{abstract}

S. Thiriot

TAO Project, INRIA Saclay, France, e-mail: samuel.thirioteres-ear.ch

Z. Lewkovicz

LIP6, Universite Paris VI, France e-mail: zach. lewkovicz@lip6. fr

P. Caillou

LRI, Universite Paris Sud, France e-mail: caillou@lri.fr

J.-D. Kant

LIP6, Universite Paris VI, France e-mail: kantelip6.fr 


\section{Introduction}

Field studies on job search highlighted several stylized facts. First, Stylized Fact 1 [SF1] : searching and finding a job implies the use of social acquaintances to retrieve information (see [8] for a review). This social structure is commonly represented using the social network metaphor: each individual is represented as a node, and communication links as edges in this network. From an economic perspective, the communication of job opportunities through social relationships may lead to incomplete information in the market, thus possibly leading to a suboptimal market efficiency. Many recent models of the labor market already described such a social network and studied its influence on the market's efficiency (e.g. [9, 4]).

Secondly, [SF2] : all ties (links) are not equally useful for job seekers, nor lead to the same information. Since the famous Granovetter's studies on job search [6, 7], it became common to distinguish weak and strong ties; strong ties in a social network reflect frequent interactions between individuals, while weak ties typically lead to less frequent and less personal relationships. Until now, only few models (notably [11]) described several link types to study the impact of this stylized fact on the market.

Moreover, strong ties are more local, because they are mainly created and maintained because of common workplaces (co-workers), life-places or other activities (near family, friends); typically, the clusters observed in social networks are mainly made of strong ties. Weak ties are more random in the network; they correspond to old friendships born at school or for family. Granovetter observed that despite of long distance and rare interactions, weak ties are more efficient for finding job opportunities than strong ties: strong ties correspond to less diversified people who may communicate easily but receive the very same information, while weak ties link more different people exposed to different types of information. These observations were replicated on different countries and populations (see [12, p.5] and [8] for detailed reviews).

Thirdly, [SF3] shared by all empirical studies on social networks [5, 15], underlines the complex nature of these social networks: the positions of agents in their social environment is far from being random; at the dyadic level, it appears that people tend to bond together when they have close socio-demographic characteristics or interests (homophily), or more generally that the existence of a social tie depends on the properties of individuals (assortativness). Some recent models included this kind of complex properties such as homophily on employment status [3] or ethnicity [12] (see also [8] for a detailed review of existing models). Nevertheless, because of the difficulty to generate plausible networks ${ }^{1}$ including individuals with several

\footnotetext{
${ }^{1}$ We define "plausible networks" as networks which, given state-of-art metrics and observation tools, complies with our limited knowledge on the real networks.
} 
interdependent attributes ${ }^{2}$, these "more detailed" networks remain very simplified compared to real ones.

The use of social acquaintances to search for jobs often changes with location and demographic characteristics. Living in the same location increases the probability of co-working, as do similar socio-demographic characteristics [2]. Moreover, complex patterns are robustly observed in real networks at the scale of the triad (strong clustering or transitivity rate, intuitively corresponding to the "friends of my friends are also my friends" effect). The recent stream of statistical analysis of large networks [10] also highlighted network-scale properties of real networks, including the frequent presence of biased distribution of degree of connectivity (most people have few ties, while few trust a big number of relationships).

The numerous studies listed before suggest a strong impact of the structure of social network on the efficiency of job search, including the strong impact of specific properties of networks on this market ${ }^{3}$.

Previous studies focus either on the impact of initial networks detailed in various levels on the markets' dynamics or on the strategic evolution of simple networks. In this paper, we describe both a rich initial network and its evolution. Among others, this approach aims to provide possible answers to the question: "how realistic initial networks should be in labor market models where these networks evolve endogenously ?". In order to simplify the generation of "rich" networks, based on properties (e.g. assortativness, homophily or communities) matching those of real networks, we use an independent software generator dedicated to the reconstruction of plausible networks for social simulation. This original experimental setting enables to explore easily the impact of different networks.

In the next section we will describe the two components of our experimental setting: the use of an generic network generator for constructing the initial networks and a simple model of the labour market. In section 3 we show some results of experiments focused on the efficiency of various link types for finding a job, and on the evolution of networks.

\footnotetext{
${ }^{2}$ For instance, let us suppose we wish to generate a multiplex network containing strong and weak ties, with agents being tagged with a "red" or "blue" color supposed to reflect their ethnicity, and a "gender" attribute that influences their friendship links. Should we first generate the network and then set the color attribute of agents in order to reflect the homophily observed from field studies, as done in [12] ? Once this first attribute is set, how could we assign gender attributes in a plausible way ? The addition of each supplementory attribute requires new algorithms, thus making the exploration of the sensitivity of markets to rich networks time-costly and hard to reproduce and communicate. In short, this technical limitation on networks' generation makes almost impossible the computationnal study of the impact of numerous complex properties which are both observed in real networks and presumed to impact job search.

${ }^{3}$ Note that some authors used networks, not for describing the structure of communications, but rather to analyze patterns of employment by describing them as networks [13].
} 


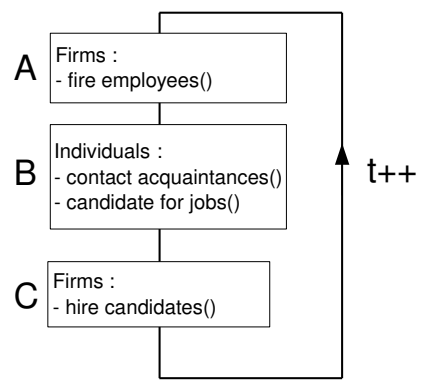

Fig. 1 A whole cycle in the simulation

\section{Model and Experimental settings}

\subsection{Labor market model}

We describe a basic multi-agent model of the labor market, in which agents represent both the firms (characterized by a number of jobs) and individuals (employed or unemployed). This market is designed such that the total number of individuals $N_{i}$ is proportional to the total number of jobs $N_{j}$ :

MarketTension $=\frac{N_{i}}{N_{j}}$.

The social network describes the structure of communication used by the agents to communicate job opportunities. Tacking stock of empirical studies (see before), we describe different kinds of relationships in this multiplex network: friendship links, colleagues (including both current colleagues and former ones) and spouses.

The behaviour of an agent, ran at each step, depends of his employment status.

A cycle in the simulation takes place in 3 parts (Figure 1). First (A), Firms randomly lay off some of their employees with a random probability FiringProba. Otherwise the employee is kept in the Firm.

In the second part (B) Individual agents interact. If they are unemployed, they look for a job. In this model, individuals may only find jobs using their social acquaintances (as done previously [1]). The Individual agents may contact their friends, colleagues etc. In order to comply with [SF2], a probability of interaction $p$ (linktype) is associated to each link type in order to communicate positions. Then they candidate to all the vacancies they are aware of.

In the last part of the cycle (C) Firms iterate all their vacancies. If a vacancy has no candidature requests, it stays vacant. When a vacancy has several candidates, the Firm chooses randomly a candidate to be hired under the condition that it will not hire an individual that it has just laid off. As soon as an individual is hired, his new colleagues are added to his set of "colleague" acquaintances; however, in order to forbid agents to know the entire population, colleagues are them removed randomly from this list of acquaintances, in order to keep the list of colleagues 
to at most max_colleagues (parameter). As a consequence, individuals remember past colleagues from past positions; however, the older the colleague, the higher the probability to break this tie.

\subsection{Social network generation}

We represent as a social network the social relationships that may lead to the communication of job offers. In this network, vertices are agents (either individuals or firms) while edges represent a dyadic relationship between these agents.

Networks used as parameters are built with the YANG ${ }^{4}$ network generator [14] which stands as a generic tool dedicated to the generation of plausible networks for social simulation. This flexible tool enables the tuning of the networks' properties, including the definition of dense clusters of colleagues and the random creation of friendships links across the network. Throughout experiments, the size of firms (number of jobs) or the number of friends will be tuned for assessing their impact on the simulation.

The network generator accepts as many discrete agents' attributes as desired. Attributes of agents are described in YANG as random variables in a Bayesian network. This formalism enables the description of interdependencies between attributes. Probabilities associated with these variables are defined as follows: agentTypes takes value 'firm' with probability 0.1 and 'individual' with probability 0.9 , leading the generator to create one firm per nine individuals. In the same way, gender take 'male' and 'female' values with probability 0.5 for individuals and value 'notRelevant' for firms. At initialization, $10 \%$ of the workers are not tied to firms and will have to find a job ${ }^{5}$. In practice, the degree for friendship (attribute auto_friends_degree) will be set to 5 or 2 , depending to the experiments. In and out degree of connectivity for the matching of firms (auto_eco_indegree and auto_eco_outdegree) respectively describe the number of links getting out of an individual (1 if employed, 0 else) and going in a firm ( 9 for all firms in the first experiments).

The last parameters of the generator are the generation rules ${ }^{6}$, which describe how the links are actually created in the population. YANG accepts two types of generation rules: "attributes rules" refer to generation rules that match two agents depending to their attributes, while "transitivity rules" propose the creation of links at the triadic scale by transitivity. We define the generation rules described in Table 1. The spirit of these rules, which will be applied in this order, is to create wedding links; then, to attribute to each worker a firm; then, to create links between all the colleagues; last, to create friendship links randomly across the population.

\footnotetext{
${ }^{4}$ http://yang.res-ear.ch

${ }^{5}$ Which will generate initially $10 \%$ of unemployment.

${ }^{6}$ Note that attributes rules always implicitly take into account the degree described before as an attribute of the agent. Laso notes that some of these rules are changed in some experiments.
} 


\begin{tabular}{|c|c|c|}
\hline rule name & method & principle \\
\hline wedding & attributes & $\begin{array}{l}\text { create links 'spouses' between males and females } \\
\text { for } 80 \% \text { of agents with max degree } 1\end{array}$ \\
\hline match & attributes & $\begin{array}{l}\text { create links 'worksInFirm' between individuals } \\
\text { having 'employed' as salarialStatus and firms }\end{array}$ \\
\hline colleagues & transitivity & $\begin{array}{l}\text { when an agent } \mathrm{A} 1 \text { 'worksInFirm' } \mathrm{A} 2 \text {, and an- } \\
\text { other agent } \mathrm{A} 3 \text { 'worksInFirm' } \mathrm{A} 2 \text {, then create a } \\
\text { link 'colleague' } \mathrm{A} 1 \text { and } \mathrm{A} 3\end{array}$ \\
\hline \multicolumn{2}{|c|}{ friendsRandom attributes } & $\begin{array}{l}\text { create links 'friendship' between individuals in } \\
\text { pure random way }\end{array}$ \\
\hline
\end{tabular}

Table 1 Generation rules

The YANG network generator uses all of these parameters for generating random networks of size N. It first creates the whole population, each agent being given a combination of the possible attributes values. This population is stored in an SQL database. Then, the generator applies all the generation rules, by retrieving agents that may be tied together by SQL set operations on the population. The software that implements the generator also provides dynamic visualization of the network generation in order to check their plausibility. More details on this algorithm are provided in [14]. The detailed parameters are provided as supplementary material for reproduction purpose.

It is important to note that, as this network generator is random, the generated population may be slightly biased; for instance, the actual proportions of agents and firms may be $85 / 15$ instead of the theoretical 90/10. As a consequence, the number of positions and individuals in sometimes not strictly equal in generated networks. To solve this problem, when networks are loaded, open positions are removed randomly if positions are too numerous, or open positions are added if workers are too numerous.

\subsection{Experimental settings}

This model was implemented in Java (1.6) under the platform Repast ${ }^{7}$. The results we present here were attained by simulations involving 1000 agents : 900 individuals and 100 firms. We measure the aggregated value for all the indicators after 300 steps on 1000 simulations.

Except when stated otherwise, the simulations' parameters are fixed arbitrarily with these values:

- max_colleagues $=5$

- marketTension $=1$

- FiringRate $\in\{0.01 ; 0.04 ; 0.07 ; 0.1\}$

- $p($ spouse $)=1.0, p($ colleagues $)=1.0$

${ }^{7} \mathrm{http} / / /$ repast.sourceforge.net 
- $p($ friend $)=$ ProbaFriend $\in\{0.2 ; 0.4 ; 0.6 ; 0.8 ; 1\}$

The observed variables are:

- The UnemployementRate, measuring the proportion of unemployed individuals divided by the total number of individuals.

- The Diameter of the graph.

- The proportion of hiring for each link type (the link type which has first informed the hired individual of the existence of the vacant position): HiringFriend,HiringSpouse and HiringColleague.

- The previous proportion has to be normalize to take into account the links probabilities and the fact that there is a different number of colleagues and friends. We define a RelativeFriendEfficiency variable, measuring the efficiency of information given by friends, compared to information given by colleagues:

RelativeFriendEfficiency $=\frac{\text { HiringFriend }}{\text { HiringColleague }} * \frac{\text { Max_colleague }}{\text { NumberOfFriends*ProbaFriend }}$

\section{Results}

\subsection{Efficiency of link types}

In this first set of experiments, we explore which links, in this model of the labor market, allow individuals to find positions when unemployed. The first simulations are run with the same number of friends and colleagues (5 each). The probability of interaction for each link type is here systematic (perfect interaction).

The unemployment rate stabilizes around a certain level whilst the agents are being fired and search for positions in other firms through their social acquaintances (see Fig. 3).

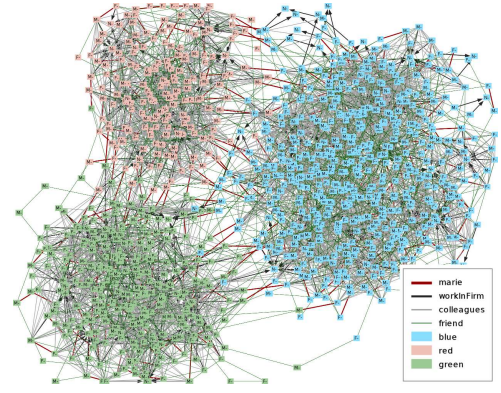

Fig. 2 Example of network used for simulations

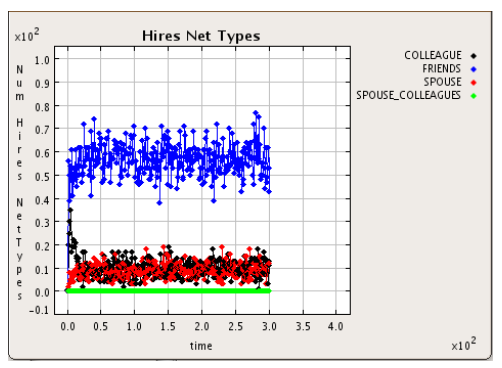

Fig. 3 Efficiency of the various link types during a typical run of the model for 5 friends and 5 colleagues 
Simulations reflect the Granovetter's strength of weak ties theory: even if individuals have the very same number of friends and colleagues, they actually find most of their positions $(\sim 55 \%)$ through friendships, which is as much as through colleague links $(\sim 34 \%)$. This effect is confirmed by other experiments. With 2 friends, the relative efficiency of friendship remains similar, each friend is $\sim 1.5$ times more useful than a colleague (see Table 2). This unemployment rate remains also similar (slightly higher) when heterogeneity is introduced into the size of firms.

\begin{tabular}{l|c|ccc|c} 
parameters & $\begin{array}{c}\text { Unemployment } \\
\text { rate }\end{array}$ & $\begin{array}{c}\text { links efficiency } \\
\text { colleagues friends spouse }\end{array}$ & $\begin{array}{c}\text { Friend vs Colleague } \\
\text { ReliveFriendEf ficiency }\end{array}$ \\
\hline same size for firms \\
\hline 5 friends & $1.8 \%$ & $37.1 \%$ & $54.5 \%$ & $8.4 \%$ & 1.48 \\
2 friends & $3.2 \%$ & $54.5 \%$ & $33.0 \%$ & $12.3 \%$ & 1.54 \\
\hline fat-tailed distribution of firms' sizes & & & \\
\hline 5 friends & $2.0 \%$ & $39.3 \%$ & $51.5 \%$ & $9.3 \%$ & 1.52 \\
2 friends & $3.3 \%$ & $53.8 \%$ & $33.8 \%$ & $12.6 \%$ & 1.58 \\
\hline
\end{tabular}

Table 2 Unemployment rate and the efficiency of link types for various combinations of parameters (with 5 colleagues). By links' efficiency we mean the proportion of hires done through this link type.

\subsection{Perfect interactions, weak sensitivity to networks' structure}

Surprisingly however, the structure of the network did not appear to have a strong impact on the unemployment rate nor on the relative importance of each link type, despite of variations such as the inclusion of three communities having a large number of endogenous links (Fig. 2). More examples with networks like the one depicted in Fig. 4 did not shift the unemployment rate as expected, despite of the strong local limitation of individuals acquaintances and of the big diameter of the graph (24 in average). These results contradict the conclusions from previous studies [3, 12] which underlined the impact of segregation on simulation results (when the network remains static during the simulation).

An analysis of the social network at different steps reveals a dramatic drop in the network size (Fig. 5), which shows how powerful the evolution of the network is, and explains the low impact of the initial network on the model. Whatever the initial properties of the network are, they will be quickly destroyed by the evolution of the network during the simulation. This may reveal a flaw in the initial model; among others, where we neglected to take into account the different possible natures of links [SF2]. These experiments led us to the idea of weighting the various types of links of interactions with probabilities (strength).

Beyond the case of labor markets, this phenomenon underlines an interesting methodological point for agent-based simulation: providing networks with many 


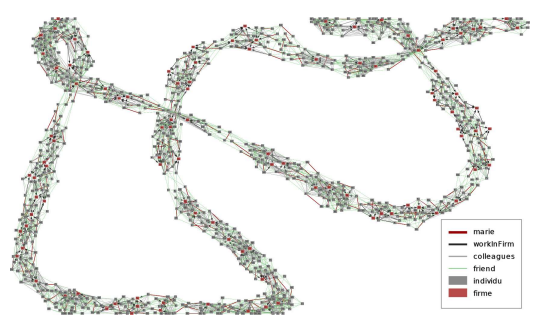

Fig. 4 Example of strongly community network used for investigating network's change during simulation

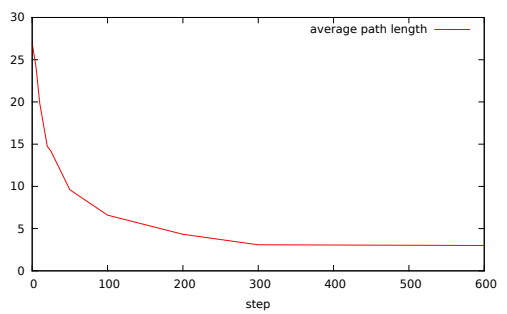

Fig. 5 Evolution of network's size during a simulation

properties - even if plausible or real - is useless if the network's dynamics is not realistic.

\subsection{Networks with probabilistic interactions}

In order to both limit this destruction of the network and enhance the plausibility of the model, we switch from a perfect to probabilistic interaction by changing the probabilities $p$ (linktype), for instance with $p$ (frienship $)=$ ProbaFriend, $p($ marie $)=1.0, p($ colleagues $)=1.0$. Given this parameters' setting, individuals have now a lower probability to discover open positions through each acquaintance, as defined in [SF2]. We can thus compare the situation with few "strong" friends configurations, with whom we communicate frequently, and more "weak" friends configurations, with less frequent interactions.

Table 3 RelativeFriendE f ficiency (relative efficiency of friend information compared to colleague information) with 5 colleagues and 5 friends. For example, when there is $10 \%$ more workers than positions (tension=1.1), when friends transmit information with probability 0.2 , each friend information transmission leads to 1.91 more hiring than a colleague information transmission.

\begin{tabular}{lrrrrrr} 
MarketTension $\backslash$ ProbaFriend & $\mathbf{0 . 2}$ & $\mathbf{0 . 4}$ & $\mathbf{0 . 6}$ & $\mathbf{0 . 8}$ & $\mathbf{1}$ Average \\
\hline 0.7 & 2.00 & 1.76 & 1.68 & 1.62 & 1.60 & 1.73 \\
0.8 & 1.96 & 1.80 & 1.65 & 1.63 & 1.58 & 1.72 \\
0.9 & 1.98 & 1.71 & 1.63 & 1.56 & 1.53 & 1.68 \\
1 & 2.06 & 1.77 & 1.63 & 1.53 & 1.50 & 1.70 \\
1.1 & 1.91 & 1.68 & 1.56 & 1.51 & 1.47 & 1.62 \\
1.2 & 1.83 & 1.61 & 1.53 & 1.48 & 1.44 & 1.58 \\
1.3 & 1.75 & 1.60 & 1.47 & 1.41 & 1.40 & 1.53 \\
\hline Average & $\mathbf{1 . 9 0}$ & $\mathbf{1 . 6 8}$ & $\mathbf{1 . 5 7}$ & $\mathbf{1 . 5 1}$ & $\mathbf{1 . 4 8}$ & $\mathbf{1 . 6 3}$
\end{tabular}

Without surprise, information coming from friends is always more useful than information coming from colleagues (hiring comes more often from friend than from colleagues after normalization by number and probability). More interestingly, 
the configuration where the friends are the most useful changes with the probability of interaction. With close friends (high probability), friends are the most efficient when the market is saturated (more jobs than employees). Friend efficiency decreases when unemployment rate increases (following market tension) because friends are more likely to be unemployed than colleagues (which were employed when they were added to the acquaintances). With weak friends' links, a second and stronger effect appears: the speed of information transfer. When there is a high unemployment rate or a high vacancy rate, to wait several steps before transmitting information has a stronger impact. With high unemployment, an other unemployed person is likely to have already taken the job. And when there is a high vacancy rate (low market tension), a colleague is likely to have found another job before the friend information arrives. The situation when weak friends are the most efficient is when there is the same number of jobs and workers.

This property is confirmed by the analysis of the RelativeFriendEfficiency sensibility toward the FiringRate parameter (the probability for each employee to be fired). Friends are more efficient than colleagues when the market is stable (low firing rate), which is understandable because the colleagues stay in the same company, and their information is thus more redundant. What is more interesting is to see that this effect is stronger for weak friends $(+55 \%$ relative efficiency from 0.1 to 0.01 firing rate) than for close friends (+20\%). Again, the time effect explains this difference: weak friend are more useful when a delay in the information has less impact (when the market is stable). Extreme cases can illustrate this: With FiringRate $=0.01$ and MarketTension $=1$, weak friends (ProbaFriend $=0.2$ ) are 2.89 times more useful than colleagues, whereas this value is only 1.47 with MarketTension $=1.3$ and FiringRate $=0.1$. Close friends efficiency even drops to 1.19 with this configuration (colleagues are almost as useful as friends when the market is saturated and changes very quickly).

Table 4 RelativeFriendEfficiency (relative efficiency of friend information compared to colleague information) with 5 colleagues and 5 friends. For example, with a probability to be fired for each worker of $1 \%$, when friends transmit information with probability 0.2 , each friend information transmission leads to 2.49 more hiring than a colleague information transmission.

\begin{tabular}{lrrrrrr} 
FiringRate $\backslash$ ProbaFriend & $\mathbf{0 . 2}$ & $\mathbf{0 . 4}$ & $\mathbf{0 . 6}$ & $\mathbf{0 . 8}$ & \multicolumn{1}{c}{ Average } \\
\hline 0.01 & 2.49 & 2.03 & 1.83 & 1.73 & 1.66 & 1.95 \\
0.04 & 1.83 & 1.63 & 1.54 & 1.47 & 1.46 & 1.58 \\
0.07 & 1.67 & 1.55 & 1.48 & 1.44 & 1.42 & 1.51 \\
0.1 & 1.61 & 1.51 & 1.44 & 1.41 & 1.38 & 1.47 \\
\hline Average & $\mathbf{1 . 9 0}$ & $\mathbf{1 . 6 8}$ & $\mathbf{1 . 5 7}$ & $\mathbf{1 . 5 1}$ & $\mathbf{1 . 4 8}$ & $\mathbf{1 . 6 3}$
\end{tabular}




\section{Discussion}

In this paper, we assess the impact of a rich network on a labor market, whose network is partly evolving when hiring employees. This study is based on an original experimental setting coupling a generic network generator and a model of the labor market. Contrary to our initial expectations, first simulations had a weak sensitivity to the initial network; we explained this fact by a fast and unrealistic evolution of the network that quickly destroyed the initial structure. Further studies with probabilistic interaction, attributed to link types, enhance the plausibility of the model and limits the evolution of the network. Further experiments highlight the impact of various properties of the initial network. For example, experiments show that, whereas friend are always more useful than colleagues, weak friends are especially useful when the market is stable and with low unemployment and vacancy rate. These experiments suggest that when the evolution of the network is not too simplistic, the description of attributes of agents and various link types in the initial network has a strong impact on the dynamics of the labor market.

\section{References}

[1] Arcaute E, Vassilvitskii S (2009) Social networks and stable matchings in the job market. Internet and Network Economics pp 220-231

[2] Bayer P, Ross S, Topa G (2005) Place of work and place of residence: Informal hiring networks and labor market outcomes. Tech. rep., NBER working paper

[3] Bramoullé Y, Saint-Paul G (2010) Social networks and labor market transitions. Labour Economics 17(1):188-195

[4] Cahuc P, Fontaine F (2009) On the efficiency of job search with social networks. Journal of Public Economic Theory 11(3):411-439

[5] Carrington PJ, Scott J, Wasserman S (2005) Models and Methods in Social Network Analysis. Cambridge University Press

[6] Granovetter M (1973) The Strength of Weak Ties. The American Journal of Sociology 78(6):1360-1380

[7] Granovetter M (1983) The strength of weak ties: A network theory revisited. Sociological Theory 1:201-233

[8] Ioannides Y, Loury D (2004) Job information networks, neighborhood effects, and inequality. Journal of Economic Literature 42(4):1056-1093

[9] Montgomery J (1991) Social networks and labor-market outcomes: Toward an economic analysis. The American economic review 81(5):1408-1418

[10] Newman M, Barabasi AL, Watts DJ (2006) The Structure and Dynamics of Networks: (Princeton Studies in Complexity). Princeton University Press, Princeton, NJ, USA

[11] Tassier T (2006) Labor market implications of weak ties. Southern Economic Journal 72(3):704-719 
[12] Tassier T, Menczer F (2008) Social network structure, segregation, and equality in a labor market with referral hiring. Journal of Economic Behavior \& Organization 66(3-4):514-528

[13] Tesfatsion L (2001) Structure, behavior, and market power in an evolutionary labor market with adaptive search* 1 . Journal of Economic Dynamics and Control 25(3-4):419-457

[14] Thiriot S, Kant JD (2008) Generate country-scale networks of interaction from scattered statistics. In: The Fifth Conference of the European Social Simulation Association, Brescia, Italy

[15] Wasserman S, Faust K (1994) Social network analysis, methods and applications. Cambridge: Cambridge University Press 